\title{
Flow cytometry in assessment of sperm integrity and functionality - a review
}

\author{
Michal Dolník ${ }^{1}$, Dagmar Mudroňová2 ${ }^{2}$ Ján Pošivák $^{1}$, Gabriel Lazar $^{1}$, Pavol Mudron̆ $^{1}$ \\ University of Veterinary Medicine and Pharmacy in Košice, ${ }^{1}$ Clinic of Ruminants, \\ ${ }^{2}$ Institute of Immunology, Košice, Slovakia
}

Received April 12, 2018

Accepted April 23, 2019

\begin{abstract}
Spermatozoa must possess many attributes to fertilize an egg but few laboratory methods can assess all of these attributes simultaneously and objectively. Most laboratory methods used to assess the quality of semen in veterinary andrology can be inaccurate and time-consuming. Laboratory techniques which evaluate only one sperm attribute, often provide results that have a weak correlation with fertility. Therefore, semen used for artificial insemination should not be assessed based on the results of one method only but rather on the comprehensive results of several laboratory tests. Flow cytometry is a modern method of analysing diJ169fferent types of cells, including sperm cells. It is based on the scattering of light and fluorescence, the outcome of which yields results that are recorded and evaluated by computer technology which makes an objective assessment. Flow cytometry in combination with fluorescence microscopy and fluorescent probes provides a comprehensive, accurate, objective, and rapid analysis of the ejaculate. In a short time frame it allows us to test thousands of sperm for their structure and properties, even with a minimal amount of semen. This method enables the evaluation of several indicators simultaneously in a population of sperm as a whole or for each sperm individually. It informs us about the selected indicators of sperm quality in the sample by examining the membrane integrity, DNA, mitochondria, acrosome, oxidative stress, and other properties. Flow cytometry has become an important method of evaluating the functional and morphological properties of sperm and is used for research in veterinary science as well as for a routine assessment of the semen quality.
\end{abstract}

Spermatozoa, fluorescent dyes, multicolour analysis, semen analysis, fertility

The development of cytometric applications in veterinary andrology has been delayed compared to the development of cytometric technologies in other disciplines and even in comparison with specialized fields in veterinary medicine (e.g., veterinary immunology). Flow cytometry dates back almost to 1970 s. It was supported by the development of microscopic analysis and the possibility of using fluorescent probes to facilitate the differentiation of specific cell populations from other cells (Karabinus et al. 1990). For many years, simple microscopic examinations have been used for sperm analysis in human and veterinary medicine. All these investigations were based on subjective evaluation (Petrunkina and Harrison 2011). Adaptation of flow cytometry for sperm analysis began as a method of measuring DNA content (Evenson et al. 1980). Currently, it can be applied to sperm count, the assessment of viability, acrosomal response, capacity status, chromatin status, mitochondrial status, oxidative stress, fluidity and membrane integrity (Mahfouz et al. 2009).

Sperm cells are complicated cells that must meet a number of criteria in order to achieve fertilization. Any disturbance of their morphology may cause them to fail in fertilization (Gillan et al. 2005). Using the knowledge of the anatomical and chemical composition of the sperm and by using suitable dyes that can penetrate into the respective sperm compartments, we are able to colourfully visualize and analyse these sections (Martí et al. 2006).

The main objective of all semen analyses is accurate, objective, quick, and inexpensive assessment of the fertilizing ability of sperm cells. Unfortunately, commonly used

Address for correspondence:

Doc. MVDr. Dagmar Mudroňová, PhD.

Institute of Immunology

University of Veterinary Medicine and Pharmacy in Košice

Komenského 73, 04181, Košice, Slovakia

Phone: +421915986954

E-mail: dagmar.mudronova@uvlf.sk

http://actavet.vfu.cz/ 
laboratory methods often fail to meet all these criteria (Gillan et al. 2005). Only a few hundred sperms or less can be examined by normal techniques at the same time. Using flow cytometry, several thousand sperm cells can be examined at the same time, wherein a matter of seconds each sperm cell is analysed individually. The use of fluorescent dyes enables simultaneous analysis of several indices relating to one sperm (membrane integrity, DNA content, mitochondrial section activity, etc.), with each indicator describing a different sperm characteristic related to its fertilizing ability or resistance to stress (Kalina et al. 2012; Peňa 2015). At the same time, the semen is analysed as a whole and based on this analysis individual sperm populations can be distinguished, e.g., sperm cells with damaged cytoplasmic membranes from sperm cells with undamaged cytoplasmic membranes (Celeghini et al. 2010). Some routinely used colour combinations and limitations of their use in flow cytometry have been well described by Peňa (2015) and Cunha et al. (2015). One of the traditional combinations of fluorescent dyes used for routine veterinary assessment of sperm viability is combination of permeable dye crossing the intact cell membrane SYBR-14 and propidium iodide which is able to enter only cells with damaged membranes. In addition to the viability assessment, determining the number of sperm cells is essential for a basic evaluation of quality which can be quickly done on the flow cytometer by counting beads (Dolník 2018). Nevertheless, the use of microscopy for the examination of sperm motility and morphological malformations is necessary.

The aim of this review was to summarize the available information about the possibilities and advantages of flow cytometry in the evaluation of qualitative and quantitative characteristics of sperm.

\section{Fluorescent probes}

The use of fluorescent probes in flow cytometry analyses is a common and also very important method for analysing the characteristics of individual cells in a mixed population (Futamura et al. 2015). Fluorescent probes are markers used to detect the expression of proteins and nucleic acids that can absorb light energy (e.g. laser) of a certain wavelength and emit light with a longer wavelength. These two processes are called excitation and emission. The emission process is routinely very fast and is known as fluorescence, which can be recorded and analysed (Aghaeepour et al. 2013). Some fluorochromes bind to specific cell molecules or components (nucleic acids, proteins, lipids) and enhance their fluorescence. Others accumulate in certain parts of cells or modify their properties through specific biochemical reactions. After the light beam passes, they visualize the particular compartment of the sperm they are bound to, thereby enabling its analysis (Silva et al. 2012).

The choice of suitable fluorescent dyes and the number of them used per one measurement depends on the flow cytometer, the excitation wavelength available on the flow cytometer, and the properties we want to analyse (Chan et al. 2012). Since other substances (impurities) may be present in the ejaculate, different contrasting dyes can be used to uniquely highlight and distinguish sperm cells. One of the most commonly used is the 4',6-diamidino-2-phenylindole dihydrochloride (DAPI), that stains the DNA of all sperm cells regardless of their viability, which is reflected by blue-coloured sperm heads under the fluorescence microscope (Gasol et al. 1999).

The discovery of various fluorochromes and fluorescent probes has allowed a broader analysis of sperm quality at biochemical, ultrastructural, and functional levels. However, a microscopic analysis alone only allows for a small number of sperm cells to be measured from the population, and additionally is time-consuming, subjective, and individual attributes must be evaluated individually. By contrast, analysis of semen by flow cytometry can ensure rapid and accurate measurement of multiple sperm characteristics necessary to achieve fertilization (Gillan et al. 2005). 


\section{Sperm viability assessment}

Within this analysis, living sperm cells are defined as sperm cells with an undamaged cytoplasmic membrane. Therefore, the integrity of the plasma membrane can be considered an indirect indicator of sperm viability. We can perform the analysis using fluorescent dyes that stain either living or dead sperm cells, or a combination of both dyes (Gillan et al. 2005). The most commonly used dye for staining dead sperm cells is propidium iodide (PI). It can pass through damaged membranes and colour the DNA to red (Oldenhof et al. 2011). It is often combined with some vital dyes such as carboxyfluorescein diacetate (CFDA) or SYBR-14, which are seen as structures stained with green fluorescence in living sperm cells. The CFDA staining principle is based on the ability of nonfluorescent CFDA to penetrate into cells, however only in viable, metabolically active cells, it can be cleaved by means of enzymes into fluorescing carboxyfluorescein (cF) (Dolník 2018). This combination of fluorescent dyes allows us to distinguish between three types of cells: 1) living sperm cells emitting green fluorescence as a result of positive staining with CFDA or SYBR-14; 2) dead sperm cells emitting red fluorescence by positive staining with PI; and 3) damaged sperm cells emitting fluorescence of both dyes (Magistrini et al. 1997) (Plate II, Fig. 1).

Using flow cytometry we can also determine the number of sperm cells. Determining the numbers of sperm cells is important in order to properly dilute the semen and create insemination doses with the desired sperm concentration. It also informs us about the production ability of the testes. The most accurate number can be determined using commercially available fluorescent counting particles. Using these, it is possible to determine the total number of sperm cells in the sample by flow cytometry simultaneously with the analysis of other properties required for fertilization (Hos sain et al. 2011).

Flow cytometry also enables us to distinguish between sperm cells killed by the necrotic process and sperm cells killed by programmed cell death (apoptosis), which is of major importance for the fertilizing ability of sperm cells (Martí et al. 2006). In this test, fluorescently labelled annexin V is used in combination with PI (Plate II, Fig. 2). During apoptosis, phosphatidylserine is translocated from the inner side to the outside of the cytoplasmic membrane, where annexin V binds to it (Anzar et al. 2002).

With flow cytometry, it is possible to assess the integrity of the entire cytoplasmic membrane, which is necessary to maintain the stability of the internal environment (Rodríguez-Martínez 2003), or of the acrosome which is necessary for egg fertilization (Hossain et al. 2011).

\section{Determination of acrosome integrity}

The acrosome is a membrane-enclosed structure covering the front part of the sperm head. It contains enzymes necessary for the penetration of the eggshells, making it an important factor for fertilization. Acrosome integrity can be analysed by a number of methods. The most commonly used method is based on the use of fluorescently labelled plant lectins (Martínez-Pastor et al. 2010). The Pisum sativum agglutinin (PSA) is a pea lectin which binds to the mannose and galactose groups in the acrosomal matrix. It cannot pass through an undamaged acrosomal membrane, thus only the acrosomes of damaged sperm cells are coloured ( $\mathrm{Nagy}$ et al. 2003). Its disadvantage is its affinity to egg yolk, which is used as part of the sperm diluents and non-specifically binds to the surface of sperm cells. As a result, the acrosomal status may be evaluated incorrectly (Lybaert et al. 2009). The most popular lectin used to assess the integrity of the acrosome is Arachis hypogaea agglutinin (PNA). It is lecithin of peanut seeds that binds to galactose groups on the outer acrosomal membrane and exhibits low nonspecific binding strength to other parts of the sperm cells (Yi et al. 2012). 


\section{Assessing mitochondrial activity}

Mitochondria are very important organelles that provide the primary energy substrates for the movement of sperm cells. Their visualization uses dyes that penetrate living sperm cells and stain active mitochondria (Garner et al. 1994). One of the first dyes used was Rhodamine 123 (R123). It accumulates in mitochondria where it emits green fluorescence. The intensity of fluorescence depends on the number of functional mitochondria. The disadvantages of this dye are its low sensitivity and that the dye fades after the loss of the membrane potential (Hossain et al. 2011). The most popular probe for determining mitochondrial activity is 5,5,6,6-tetrachloro-1,1,3,3-tetraethylbenzimidazolylcarbocyanine iodide (JC-1). This probe also enables the differentiation of sperm cells whereby reduced function sperm cells are visualised by green fluorescent mitochondria, whereas sperm cells with highly active mitochondria are visualised by red fluorescent clusters due to the accumulation of dye (Kasai et al. 2002). However, dual fluorescence is also a disadvantage of this dye, as two detectors are needed to assess one indicator and this also limits its use in combination with other dyes emitting red and green fluorescence (Vincent et al. 2012). MitoTracker dyes represent a group of recently developed dyes. These dyes stain active mitochondria. They are highly specific, available in a wide range of excitation and emission fluorescences and can be combined with probes requiring fixation (Martinéz-Pastor et al. 2010). They were successfully used in a multicolour analysis (García-Álvarez et al. 2009).

\section{Sperm chromatin analysis}

Examination of DNA integrity is of the highest importance for normal embryo development. Although DNA is packed in a highly compact and stable form, it can be damaged and chromatin abnormalities can occur (Carrell et al. 2003). The origin of these changes can be caused by intra- and extra-testicular conditions. During their development, sperm cells are susceptible to both exogenous and endogenous stressors that can affect them during spermatogenesis, when structural changes occur in chromatin, or during sperm maturation in the epididymis. Damage of chromatin may also result from free radicals or apoptosis (Haines et al. 2002). Due to the importance of accurate transmission of genetic information to the offspring, several methods have been developed to detect damaged DNA in sperm and there has been an increased effort to integrate these tests into routine semen analyses (Cordelli et al. 2005).

The sperm chromatin structure assay (SCSA) uses an acridine orange dye and is based on high sensitivity of abnormal chromatin to induced DNA denaturation. In the denaturation process, damaged DNA is untwisted. In the next step, the dual fluorescence ability of acridine orange is utilized, while the untwisted, single-stranded DNA is stained red and double-stranded DNA is stained green (Evenson and Wix on 2006). The SCSA results show the extent of DNA denaturation as a DNA fragmentation index, which is the ratio of red fluorescence to total fluorescence intensity. A high value in this index correlates to high DNA damage (Giwercman et al. 2010). This method is limited by the possible incompatibility of the denaturation solution with some other probes as well as the double fluorescence of acridine orange, which reduces the possible use of other probes (Vincent et al. 2012).

Fragmentation of sperm DNA can be determined using TUNEL (terminal transferased UTP nick-end labelling) test. Using this test, we can identify breaks in the DNA chain on which they are located due to damage on the free $3-\mathrm{OH}$ ends. These $3-\mathrm{OH}$ ends of DNA fragments can be visualized by fluorescently labelled nucleotides under catalysis by the terminal deoxynucleotidyl transferase enzyme. Incorporated labelled nucleotides can subsequently be detected in the sperm using light microscopy, fluorescence microscopy or flow cytometry (Petrunkina and Harrison 2011). This method makes it possible to 
quantify the number of sperm cells with damaged DNA, but also to determine the degree of damage based on fluorescence. The intensity of fluorescence increases with an increase in the number of free 3-OH ends, which is in direct correlation with the degree of sperm damage (Vincent et al. 2012). TUNEL test is a promising method that demonstrates a good mutual relationship with SCSA test as well as with fertility (Benchaib et al. 2007). TUNEL test can be used in flow cytometry, although Dominguez-Fandos et al. (2007) reported $2.6 \times$ higher sperm damage in flow cytometry compared to fluorescence microscopy.

\section{Detection of oxidative stress and lipoperoxidation by flow cytometry}

There are a number of direct and indirect methods available for determining reactive oxygen species (ROS) but direct determination is very challenging due to the instability of ROS and the short life of their intermediates. Oxidative stress analysis using flow cytometry became available with the discovery of new fluorochromes. Various fluorescent probes that respond specifically to a particular type of ROS or non-specific probes that are oxidized by multiple types of ROS can be used. Depending on the degree of fluorescence we can establish the level of oxidative stress (Hossain et al. 2011).

An example of a specific dye is dihydroethidium (DHE) which reacts with the superoxide anion $\left(\mathrm{O}_{2}^{-}\right)$, forming yellow 2-hydroxy-ethidium at a wavelength of $567 \mathrm{~nm}$ (Zhao et al. 2005). However, it is clear from the works of other authors that other types of ROS can be detected using other filters (Burnaugh et al. 2007). Another specific probe is MitoSOXTM ${ }^{\mathrm{TM}}$ (Thermo Fisher, Waltham, MA USA). This probe is able to detect mitochondrial superoxides in populations of living sperm cells (Koppers et al. 2008).

One non-specific dye is 2,7-dichlorodihydrofluorescein diacetate ( $\left.\mathrm{H}_{2} \mathrm{DCFDA}\right)$ which serves as an indicator of the intracellular presence of ROS in sperm cells (Kim et al. 2010). $\mathrm{H}_{2}$ DCFDA passes through the plasma membrane and is retained after intracellular cleavage by esterases, and emits green fluorescence as a result of oxidation (Martinez-Pastor et al. 2010).

Oxidative attack on membranes results in lipoperoxidation which has a negative effect on sperm quality. The level of lipoperoxidation was successfully evaluated by flow cytometry using a C11-BODIPY581/591 probe in bull (Brouwers and Gadella 2003) and stallion (Ortega et al. 2009) semen. C11-BODIPY581/591 is a fatty acid analogue which can be incorporated into the sperm cell membrane. Its reactivity to peroxidation is comparable to the reactivity of unsaturated fatty acids which are abundant in the sperm cell membrane (Drummen et al. 2004; Dolník et al. 2018). This probe emits red fluorescence in an unoxidized state which changes to bright green after peroxidation (Christova et al. 2004). Based on the advantages and properties of this probe, as well as the high sensitivity of fluorescence techniques, it can be considered as an indicator of the exposure of phospholipids to ROS. This probe can be used simultaneously with PI. This staining enables the differentiation of the level of lipoperoxidation in the sperm cells and also the damage to them since PI can only penetrate into cells with a damaged membrane (Brouwers and Gadella 2003) (Plate III, Fig. 3).

\section{Conclusion}

Flow cytometry is a very useful method for assessing fertility in veterinary andrology. When assessing the quality of the ejaculate, the assessment of only one or two indicators does not objectively determine whether the sperm cells are able to fertilize the egg because the damage may be in another area of the sperm cells. For this reason, multiparameter analysis plays a very important role. By using various fluorescent dyes it is possible to better visualize the entire sperm cell or, alternatively, to solely visualise its individual parts. This enables us not only to separate different groups of sperm cells (e.g., living from the dead), but also to 
determine which part of the sperm cell is damaged in the case of poor fertilization ability of the sperm. Flow cytometry enables quality examination of the functional and morphological characteristics of sperm cells and also helps us to better understand the biology of fertilization.

\section{Acknowledgement}

This work was supported by Research Grant Agency of the Ministry of Education, Science, Research and Sport of the Slovak Republic (VEGA 1/0107/17).

\section{References}

Aghaeepour N, Finak G, Hoos H, Mosmann TR, Brinkman R, Gottardo R, Scheuermann RH 2013: Critical assessment of automated flow cytometry data analysis techniques. Nat Methods 10: 228-238

Anzar M, He L, Buhr MM, Kroetsch TG, Pauls KP 2002: Sperm apoptosis in fresh and cryopreserved bull semen detected by flow cytometry and its relationship with fertility. Biol Reprod 66: 354-360

Benchaib M, Lornage J, Mazoyer C, Lejeune H, Salle B, Guerin FJ 2007: Sperm deoxyribonucleic acid fragmentation as a prognostic indicator of assisted reproductive technology outcome. Fertil Steril 87: 93-100

Brouwers JF, Gadella BM 2003: In situ detection and localization of lipid peroxidation in individual bovine sperm cells. Free Radic Biol Med 35: 1382-1391

Burnaugh L, Sabeur K, Ball BA 2007: Generation of superoxide anion by equine spermatozoa as detected by dihydroethidium. Theriogenology 67: 580-589

Carrell DT, Liu L, Peterson CM, Jones KP, Hatasaka H, Erickson L, Campbell B 2003: Sperm DNA fragmentation is increased in couples with unexplained recurrent pregnancy loss. Arch Androl 49: 49-55

Celeghini CCE, de Andrade CFA, Raphael FC, Nascimento J, Ticianelli SJ, de Arruda PR 2010: Damage assessment of the equine sperm membranes by fluorimetric technique. Braz Arch Biol Technol 53: 1285-1292

Chan J, Dodani SC, Chang CJ 2012: Reaction-based small-molecule fluorescent probes for chemoselective bioimaging. Nat Chem 4: 973-984

Christova Y, James PS, Jones R 2004: Lipid diffusion in sperm plasma membranes exposed to peroxidative injury from oxygen free radicals. Mol Reprod Dev 68: 365-372

Cordelli E, Eleuteri P, Leter G, Rescia M, Spanó M 2005: Flow cytometry applications in the evaluation of sperm quality: semen analysis, sperm function and DNA integrity. Contraception 72: 273-279

Cunha ATM, Carvalho JO, Dode MAN 2015: Techniques for sperm evaluation using fluorescent probes. Semin Ciênc Agrár 36: 4365-4376

Dolník M 2018: Effect of selenium on the fertilizing characteristics of ruminant spermatozoa (dissertation), $95 \mathrm{p}$.

Dolník M, Pošivák J, Kadáši M, Klein R, Lazar G, Mudroňová D 2018: Evaluation of the sperm fertilization status by flow cytometry. Magyar Állatorvosok Lapja 140: 337-341

Domínguz-Fandos D, Camejo MI, Ballesca JL, Oliva R 2007: Human sperm DNA fragmentation: correlation of TUNEL results as assessed by flow cytometry and optical microscopy. Cytometry A 71A: 1011-1018

Drummen GP, Gadella BM, Post JA, Brouwers F 2004: Mass spectrometric characterization of the oxidation of the fluorescent lipid peroxidation reporter molecule C11-BODIPY. Free Radic Biol Med 36: 1635-1644

Evenson DP, Darzynkiewicz Z, Melamed MR 1980: Comparison of human and mouse sperm chromatin structure by flow cytometry. Chromosoma 78: $225-238$

Evenson DP, Wixon R 2006: Clinical aspects of sperm DNA fragmentation detection and male infertility. Theriogenology 65: 979-991

Futamura K, Sekino M, Hata A, Ikebuchi R, Nakanishi Y, Egawa G, Kabashima K, Watanabe T, Furuki M, Tomura M 2015: Novel full-spectral flow cytometry with multiple spectrally-adjacent fluorescent proteins and fluorochromes and visualization of in vivo cellular movement. Cytometry A 87: 830-842

Garcia-Álvarez O, Maroto-Morales A, Martínez-Pastor F, Garde JJ, Ramón M, Fernández-Santos RM, Esteso CM, Pérez-Guzmán DM, Soler JA 2009: Sperm characteristics and in vitro fertilization ability of thawed spermatozoa from Black Manchega ram: Electroejaculation and postmortem collection. Theriogenology 72 : 160-168

Garner DL, Johnson LA, Yue ST, Roth BL, Haugland RP 1994: Dual DNA staining assessment of bovine sperm viability using SYBR-14 and propidium iodide. J Androl 15: 620-629

Gasol JM, Zweifel UL, Peters F, Fuhrman JA, Hagstrom A 1999: Significance of size and nucleic acid content heterogeneity as measured by flow cytometry in natural planktonic bacteria. Appl Environ Microbiol 65: 4475-4483

Gillan L, Evans G, Maxwell WM 2005: Flow cytometric evaluation of sperm parameters in relation to fertility potential. Theriogenology 63: 445-457

Giwercman A, Lindstedt L, Larsson M, Bungum M, Spano M, Levine RJ, Rylander L 2010: Sperm chromatin structure assay as an independent predictor of fertility in vivo: a case-control study. Int J Androl 33: 221-227

Haines GA, Hendry JH, Daniel CP, Morris ID 2002: Germ cell and dose-dependent DNA damage measure by the comet assay in murine spermatozoa after testicular X-irradiation. Biol Reprod 67: 854-861

Hossain MS, Johannisson A, Wallgren M, Nagy S, Siqueira AP, Rodriguez-Martinez H 2011: Flow cytometry for the assessment of animal sperm integrity and functionality: state of art. Asian J Androl 13: 406-419 
Kalina T, Flores-Montero J, van der Velden VH, Martin-Ayuso M, Bottcher S, Ritgen M, Almeida J, Lhermitte L, Asnafi V, Mendonca A, de Tute R, Cullen M, Sedek L, Vidriales MB, Pérez JJ, te Marvelde JG, Mejstrikova E, Hrusak O, Szczepański T, van Dongen JJM, Orfao A 2012: Euroflow standardization of flow cytometer instrument settings and immunophenotyping protocols. Leukemia 26: 1986-2010

Karabinus DS, Evenson DP, Jost LK, Baer RK 1990: Comparison of semen quality in young and mature Holstein bulls measured by light microscopy and flow cytometry. J Dairy Sci 73: 2364-2371

Kasai T, Ogawa K, Mizuno K, Nagai S, Uchida Y, Ohta S, Fujie M, Suzuki K, Hirata S, Hoshi K 2002: Relationship between mitochondrial membrane potential, sperm motility and fertility potential. Asian J Androl 4: 97-103

Kim SH, Yu DH, Kim YJ 2010: Apoptosis-like change, ROS, and DNA status in cryopreserved canine sperm recovered by glass wool filtration and Percoll gradient centrifugation techniques. Anim Reprod Sci 119: 106-116

Koppers, AJ, De Luliis GN, Finnie JM, McLaughlin EA, Aitken J 2008: Significance of mitochondrial reactive oxygen species in the generation of oxidative stress in spermatozoa. J Clin Endocrinol Metab 93: 3199-3207

Lybaert P, Danguy A, Leleux F, Meuris S, Lebrun P 2009: Improved methodology for the detection and quantification of the acrosome reaction in mouse spermatozoa. Histol Histopathol 24: 999-1007

Magistrini M, Guitton E, Levern Y, Nicolle ClJ, Vidament M, Kerboeuf D, Palmer E 1997: New staining methods for sperm evaluation estimated by microscopy and flow cytometry. Theriogenology 48:1229-1235

Mahfouz R, Said MT, Agarwal A 2009: The diagnostic and therapeutic applications of flow cytometry in male infertility. Arch Med Sci 5: 99-108

Martí E, Pérez-Pé R, Muino-Blanco T, Cebrián-Pérez JA 2006: Comparative study of four different sperm washing methods using apoptotic markers in ram spermatozoa. J Androl 27: 746753

Martínez-Pastor F, Mata-Campuzano M, Álvarez-Rodríguez M, Álvarez M, Anel L, de Paz P 2010: Probes and techniques for sperm evaluation by flow cytometry. Reprod Domest Anim 45: 67-78

Nagy S, Jansen J, Topper EK, Gadella BM 2003: A triple-stain flow cytometric method to assess plasma- and acrosome-membrane integrity of cryopreserved bovine sperm immediately after thawing in presence of eggyolk particles. Biol Reprod 68: 1828-1865

Oldenhof H, Blässe AK, Wolkers WF, Bollwein H, Sieme H 2011: Osmotic properties of stallion sperm subpopulations determined by simultaneous assessment of cell volume and viability. Theriogenology 76 : 386-391

Ortega FC, Gonzáles FL, Morrell JM, Salazar SC, Macias GB, Rodríguez-Martinez H, Tapia JA, Peňa FJ 2009: Lipid peroxidation, assessed with BODIPY-C11, increases after cryopreservation of stallion spermatozoa, is stallion-dependent and is related to apoptotic-like changes. Reproduction 138: 55-63

Peňa FJ 2015: Multiparametric flow cytometry: a relevant tool for sperm function evaluation. Anim Reprod 12: 351-355

Petrunkina AM, Harrison RA 2011: Cytometric solutions in veterinary andrology: developments, advantages, and limitations. Cytometry A 79: 338-348

Rodríguez-Martínez H 2003: Laboratory semen assessment and prediction of fertility: still utopia? Reprod Domest Anim 38: 312-318

Silva TL, Roseiro JC, Reis A 2012: Applications and perspectives of multi-parameter flow cytometry to microbial biofuels production processes. Trends Biotechnol 30: 225-232

Vincent P, Underwood SL, Dolbec C, Bouchard N, Kroetsch T, Blondin P 2012: Bovine semen quality control in artificial insemination centers. Anim Reprod 9: 153-165

Yi Y, Zimmerman SW, Manandhar G, Oddhiambo JF, Kennedy C, Jonáková V, Maňásková-Postlerová P, Sutovský M, Park CS, Sutovsky P 2012: Ubiquitin-activating enzyme (UBA1) is required for sperm capacitation, acrosomal exocytosis and sperm-egg coat penetration during porcine fertilization. Int J Androl 35: 196-210

Zhao H, Joseph J, Fales HM, Sokoloski EA, Levine RL, Vasquez-Vivar J, Kalyanaraman B 2005: Detection and characterization of the product of hydroethidine and intracellular superoxide by HPLC and limitations of fluorescence. Proc Natl Acad Sci USA 102: 5727-5732 
Plate II

Dolník M. et al.: Flow cytometry ... pp. 169-175
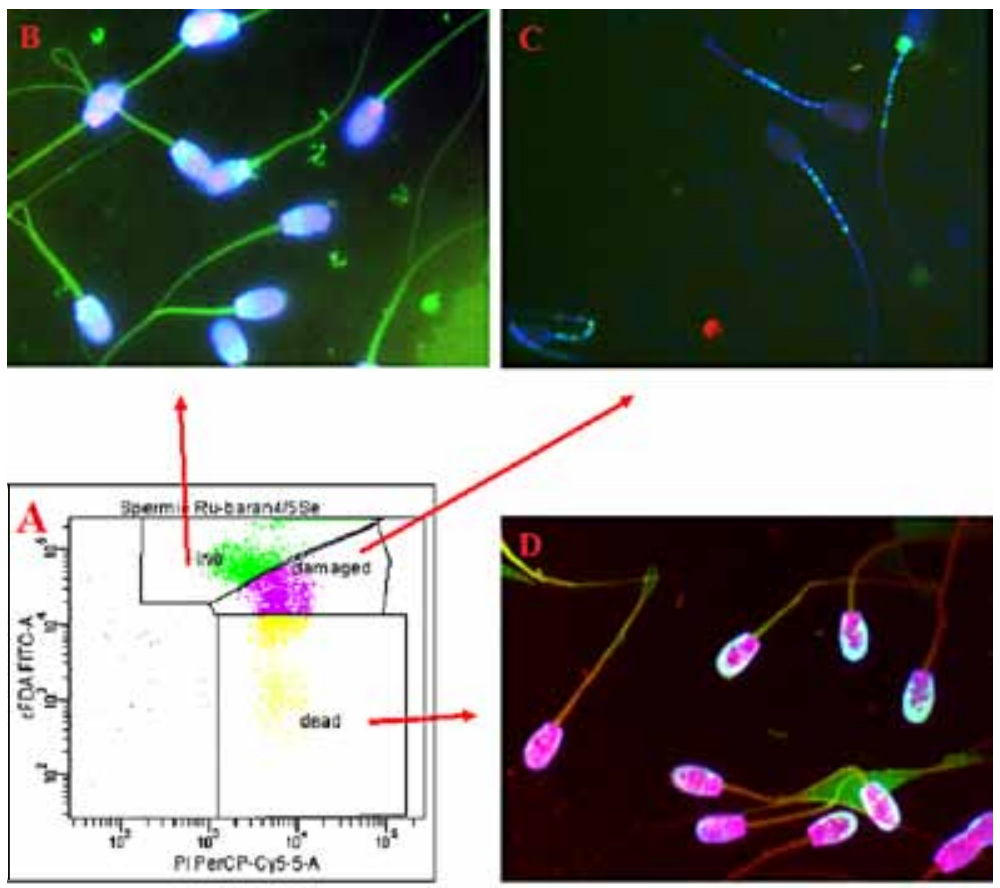

Fig. 1. Sperm viability analysis evaluated by flow cytometry and fluorescence microscopy using contrast dye DAPI and fluorescent dyes CFDA and PI. A: Dot plot from a flow cytometer. Green colour represents live sperm; yellow represents dead sperm; dying sperm are represented by purple colouration. B: Illustration of living sperm by a fluorescence microscope. Mitochondrial apparatus is stained green with CFDA. The blue colour of the head is due to the contrast dye DAPI. C: Illustration of dying sperm whose mitochondrial section is still stained by CFDA, but the heads are stained by PI and DAPI. D: Illustration of dead sperm after staining with PI. The pink colour of the heads is due to the common fluorescence of the red dye PI and the blue dye DAPI.

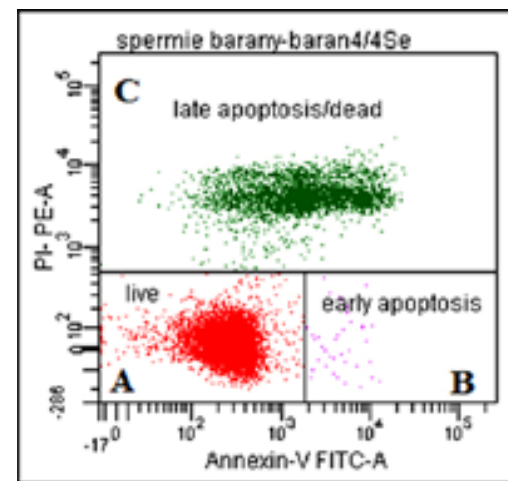

Fig. 2. Dot plot of flow cytometric sperm analysis of the level of apoptosis. A: Live sperm. B: Sperm in the early phase of apoptosis. C: Dead sperm and sperm in the late phase of apoptosis. 
Plate III

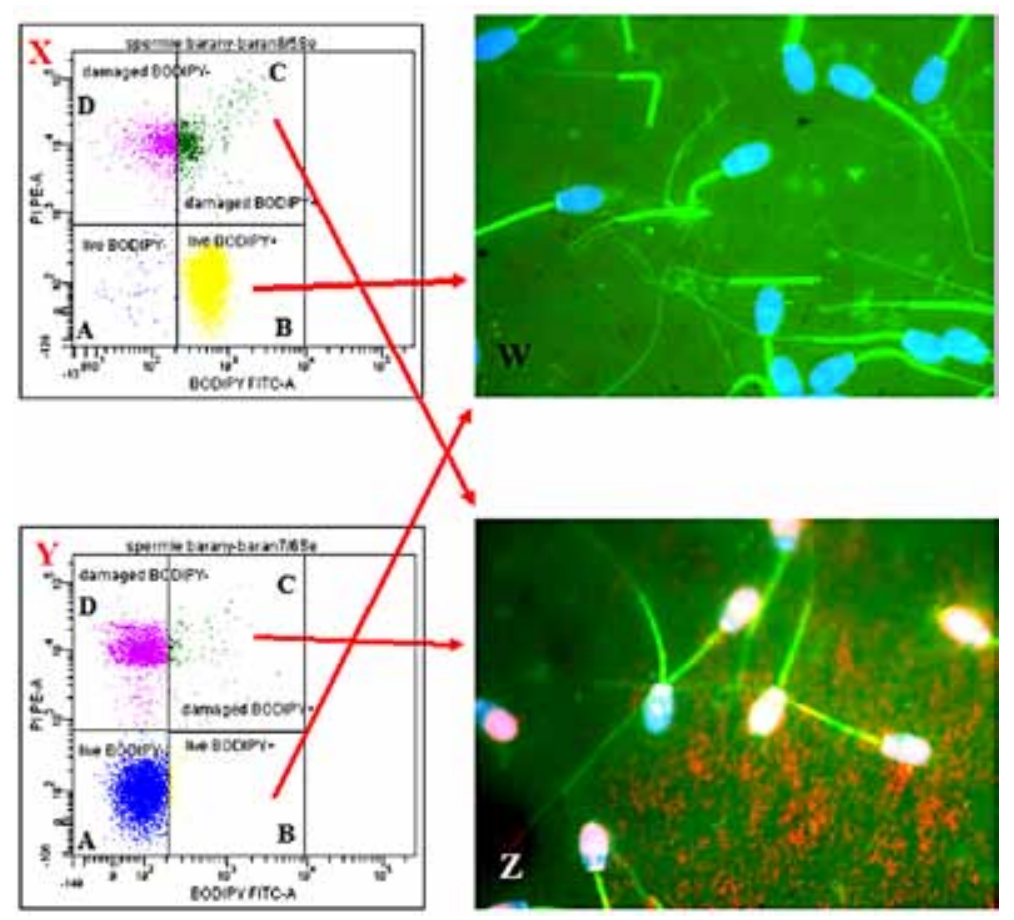

Fig. 3. Illustration of cytometric and microscopic examination of lipoperoxidation level and damaging sperm using the dye C11-BODIPY 581/591 and PI. X: Semen with high level of oxidative stress. It can be distinguished in individual quadrants of the dot plot graph. Y: High quality semen with low level of lipoperoxidation. A: Live sperm without oxidative stress (blue). B: Live sperm with oxidative stress (yellow). C: Damaged sperm with oxidative stress (green). D: Damaged sperm without oxidative stress (purple). On the images derived from the fluorescence microscope, the following is visible: W: live sperm with oxidative stress emitting green fluorescence (C11-BODIPY 581/591). Z: damaged sperm with oxidative stress emitting red (PI) and green (C11-BODIPY 581/591) fluorescence. 